\title{
Extract of Cordyceps militaris inhibits angiogenesis and suppresses tumor growth of human malignant melanoma cells
}

\author{
I. MADE WINARSA RUMA ${ }^{1 *}$, ENDY WIDYA PUTRANTO ${ }^{1 *}$, EISAKU KONDO $^{2}$, RISAYO WATANABE ${ }^{2}$, \\ KEN SAITO $^{2}$, YUSUKE INOUE ${ }^{3}$, KEN-ICHI YAMAMOTO $^{1}$, SUSUMU NAKATA ${ }^{2}$, \\ MASAJI KAIHATA ${ }^{4}$, HITOSHI MURATA ${ }^{1}$ and MASAKIYO SAKAGUCHI ${ }^{1}$ \\ ${ }^{1}$ Department of Cell Biology, Graduate School of Medicine, Dentistry and Pharmaceutical Sciences, \\ Okayama University, Okayama 700-8558; ${ }^{2}$ Division of Oncological Pathology, Aichi Cancer Center \\ Research Institute, Chikusa-ku, Nagoya 464-8681; ${ }^{3}$ Faculty of Science and Technology, Division of Molecular \\ Science, Gunma University, Kiryu, Gunma $376-8515 ;{ }^{4}$ CAITAC Corporation, Kita-ku, Okayama 700-8586, Japan
}

Received February 3, 2014; Accepted April 2, 2014

DOI: 10.3892/ijo.2014.2397

\begin{abstract}
Angiogenesis is essential for tumor development and metastasis. Among several angiogenic factors, vascular endothelial growth factor receptor (VEGF) is important for tumor-derived angiogenesis and commonly overexpressed in solid tumors. Thus, many antitumor strategies targeting VEGF have been developed to inhibit cancer angiogenesis, offering insights into the successful treatment of solid cancers. However, there are a number of issues such as harmful effects on normal vascularity in clinical trials. Taking this into consideration, we employed Cordyceps militaris as an antitumor approach due to its biological safety in vivo. The herbal medicinal mushroom Cordyceps militaris has been reported to show potential anticancer properties including anti-angiogenic capacity; however, its concrete properties have yet to be fully demonstrated. In this study, we aimed to elucidate the biological role of Cordyceps militaris extract in tumor cells, especially in regulating angiogenesis and tumor growth of a human malignant melanoma cell line. We demonstrated that
\end{abstract}

Correspondence to: Dr Masakiyo Sakaguchi, Department of Cell Biology, Graduate School of Medicine, Dentistry and Pharmaceutical Sciences, Okayama University, Okayama 700-8558, Japan

E-mail: masa-s@md.okayama-u.ac.jp

${ }^{*}$ Contributed equally

Abbreviations: VEGF, vascular endothelial growth factor; VEGFR, vascular endothelial growth factor receptor; PDGF, platelet derived growth factor; PIGF, placental growth factor; TIMP, tissue inhibitor of metalloproteinases; bFGF, basic fibroblast growth factor; GSK-3 $\beta$, glycogen synthase kinase-3 $\beta$; MM, malignant melanoma; mTOR, mammalian target of rapamycin; MAPK, mitogen-activated protein kinase; PI3K, phosphoinositide 3-kinase; ERK, extracellular signalregulated kinase; PKC- $\beta$, protein kinase $C \beta$; TGF, transforming growth factor

Key words: Cordyceps militaris, melanoma, angiogenesis, vascular endothelial growth factor receptor, Akt and GSK-3 $\beta$
Cordyceps militaris extract remarkably suppressed tumor growth via induction of apoptotic cell death in culture that links to the abrogation of VEGF production in melanoma cells. This was followed by mitigation of Akt1 and GSK-3 $\beta$ activation, while p38 $\alpha$ phosphorylation levels were increased. Extract treatment in mouse model xenografted with human melanoma cells resulted in a dramatic antitumor effect with downregulation of VEGF expression. The results suggest that suppression of tumor growth by Cordyceps militaris extract is, at least, mediated by its anti-angiogenicity and apoptosis induction capacities. Cordyceps militaris extract may be a potent antitumor herbal drug for solid tumors.

\section{Introduction}

Angiogenesis, the sprouting of new capillaries from preexisting vessels, is essential for physiological processes including embryonic development and tissue remodeling but is also involved in pathological conditions such as tumor development (1). Over $90 \%$ of solid tumors are highly angiogenic (2). Tumor angiogenesis results from an imbalance between pro-angiogenic factors such as VEGF and endogenous antiangiogenic factors such as angiostatin and endostatin (3). Tumor cells vary in their angiogenic potential associated with the amounts of bFGF and VEGF secreted $(4,5)$. In addition, it appears tumors that produce multiple angiogenic factors show increased rates of primary tumor expansion, survival and also metastasis (6-8). An angiogenic switch is considered prerequisite for cancer progression (9). Many triggers that are commonly encountered in precancerous or cancer cells, including low pO2, low $\mathrm{pH}$, hyper/hypoglycemia, hyperthermia, mechanical stress, immune/inflammatory responses and genetic mutations, can turn on this angiogenic switch $(2,10,11)$.

The central molecule in physiological and pathological angiogenesis is VEGF $(11,12)$. VEGFs are a heparin-binding family of glycoproteins, which includes VEGF-A, VEGF-B, VEGF-C, VEGF-D and PIGF (placental growth factor) (13). VEGF has an important role in inducing and maintaining angiogenesis in tumors (14). Among the VEGF family members, VEGF-A is the major player in angiogenesis that activates two 
tyrosine kinase receptors, VEGFR1 and VEGFR2, of which VEGFR2 has the most important role in VEGF-induced angiogenesis $(15,16)$. VEGF-A is overexpressed in many types of solid tumors and correlates with vascularity, grade and prognosis (17).

Malignant melanoma (MM) is the most lethal skin cancer. It is highly angiogenic, highly metastatic and resistant to treatment. In vivo murine studies have shown that aggressive melanoma cell lines express higher levels of VEGF than nonaggressive cell lines (2). Furthermore, significant increases in secretion and stromal deposition of VEGF are observed during the switch from radial to vertical growth of MM, suggesting a significant role of VEGF in cancer invasiveness (18). Therefore, downregulation of VEGF is a potential approach to combat MM progression.

Due to the essential role of VEGF in angiogenesis during cancer progression, many drugs have been developed and widely used to inhibit VEGF, such as anti-VEGF neutralizing antibody and small molecules that block the tyrosine kinase activity of VEGFRs. However, these approaches only benefit a small fraction of cancer patients as cancer cells can become refractory to VEGF inhibitors $(19,20)$. Therefore, alternative approaches that provide a better outcome but are also economically justified are needed. One such approach is the utilization of herbal drugs, such as Cordyceps militaris (C. militaris), a medicinal mushroom that is known to contain cordycepin, adenosine, exo-polysaccharides, and sterols (21-24). Among these metabolic active ingredients, cordycepin is known to have anticancer properties such as inhibition of purine metabolism, RNA/DNA synthesis, and the mTOR pathway (22). Therefore, many cordycepin analogues and synthetic ingredients have been developed for anticancer drugs. However, due to the limited availability in its natural environment and increasing demands, the price of $C$. militaris has greatly increased $(25,26)$.

Despite its many anticancer properties, it has not been determined whether $C$. militaris extract has an anti-angiogenic property in human MM cancer cells. In the present study, we evaluated the role of $C$. militaris extract for inhibition of angiogenesis and tumor growth of a human MM cancer cell line. We found that $C$. militaris extract prepared by a newly developed method remarkably suppressed the growth of tumor cells through mitigation of VEGF production in the human MM cancer cell line in vitro and in vivo.

\section{Materials and methods}

Cell lines and chemicals. The human malignant melanoma cell line MeWo (HTB-65) was purchased from American Type Culture Collection (ATCC) (Manassas, VA, USA) in 2002, and after expanded culture, $1 \times 10^{6}$ cells/tube were kept in liquid nitrogen. MeWo cells from stocks were cultured in RPMI medium (Invitrogen, Carlsbad, CA, USA) supplemented with $10 \%$ fetal bovine serum and used within five passages. C. militaris extract was kindly supplied by CAITAC Corp. (Okayama, Japan). Cordycepin was purchased from SigmaAldrich (St. Louis, MO, USA). Rabbit anti-VEGF polyclonal $\mathrm{Ab}$ (no. ab46154) and rat anti-mouse CD31 monoclonal Ab (no. DIA-310) were purchased from Abcam (Cambridge, UK) and Dianova (Hamburg, Germany), respectively.
Plasmid constructs. To construct a mammalian expression vector, the CMV promoter-intron (CMVi) from the phCMVFSRTM vector (Genlantis, San Diego, CA, USA) was inserted into the promoter-less pIDT-SMART vector (Integrated Device Technology, San Jose, CA, USA) and this was named pIDT-CMViR (27). The vector could efficiently express cargo cDNAs. In addition to GFP cDNA, human cDNAs encoding the full lengths of $\mathrm{p} 38 \alpha$, GSK3 $\beta$, PI3K (p85), and Akt1 were then each inserted into the pIDT-CMVi vector. Transient transfection of the plasmids to MeWo cells was performed using Xfect transfection reagent (Clontech Takara, Mountain View, CA, USA).

Antibody array. Screening for angiogenesis-related factors produced by MeWo cells was carried out by using the Human angiogenesis Antibody Array (R\&D Systems, Minneapolis, MN, USA). MeWo cells were treated with $C$. militaris extract or cordycepin and cultured for $48 \mathrm{~h}$ in serum-free RPMI medium. The medium incubated for the last $24 \mathrm{~h}$ was used for the assay. Extracts prepared from MeWo cells cultured with or without $C$. militaris extract for $48 \mathrm{~h}$ in serum-free RPMI medium were also analyzed using a proteome profiler array (Human Phospho-MAPK Array; R\&D Systems).

Evaluation of cell growth and apoptosis. For growth assay, MeWo cells were cultured in RPMI medium with $10 \%$ fetal bovine serum for $24 \mathrm{~h}$ prior to treatment with $1 \%, 5 \%$ or $10 \%$ C. militaris extract or with 20,100 or $200 \mu \mathrm{M}$ cordycepin. Cells were counted in triplicates at day 1 before treatment and subsequently on days 2, 3, 5 and 7 after seeding. Re-treatment was performed on days 3 and 5 for the remaining cultures after counting. Cells were detached with $0.20 \%$ trypsin-EDTA solution, stained with $0.3 \%$ trypan blue in PBS, and counted with a haemocytometer. For apoptosis assay, cells were incubated with 1,5 or $10 \%$ C. militaris extract or with 20 and $50 \mu \mathrm{M}$ cordycepin for $48 \mathrm{~h}$ before identification of apoptotic cells showing nuclear shrinkage or fragmentation by staining with Hoechst 33342 (Invitrogen) for $30 \mathrm{~min}$.

RNA extraction and real-time quantitative reverse transcription $(q R T)-P C R$. Total RNA was extracted using TriPure Isolation Reagent (Roche, Tokyo, Japan). Reverse-transcription was performed using ReverTra Ace qPCR RT Master Mix with gDNA Remover (Toyobo, Osaka, Japan). Real-time PCR was performed using FastStart SYBR Green Master (Roche, Basel, Switzerland) with specific primers for VEGF-A (tta aacgaacgtacttgcagatg, gagagatctggttcccgaaa), VEGF-B (ctg gccaccagaggaaagt, ccatgagctccacagtcaag), and GAPDH (agc cacatcgctcagacac, gccaatacgaccaaatcc) on a LightCycler 480 system II (Roche). The levels of amplicons were expressed relative to GAPDH as an internal control using the $\Delta \Delta \mathrm{Ct}$ method.

In vivo tumor growth assay. Two millions MeWo cells were subcutaneously implanted in each of 6 week-old female Balb/c nu/nu mice, and then $400 \mu \mathrm{l} C$. militaris extract was injected subcutaneously to surround the tumor (not directly injected into the tumor) every two days after the size of the tumor had reached $\sim 100 \mathrm{~mm}^{3}$. The results were compared with results for mice that were injected with saline alone as an untreated 


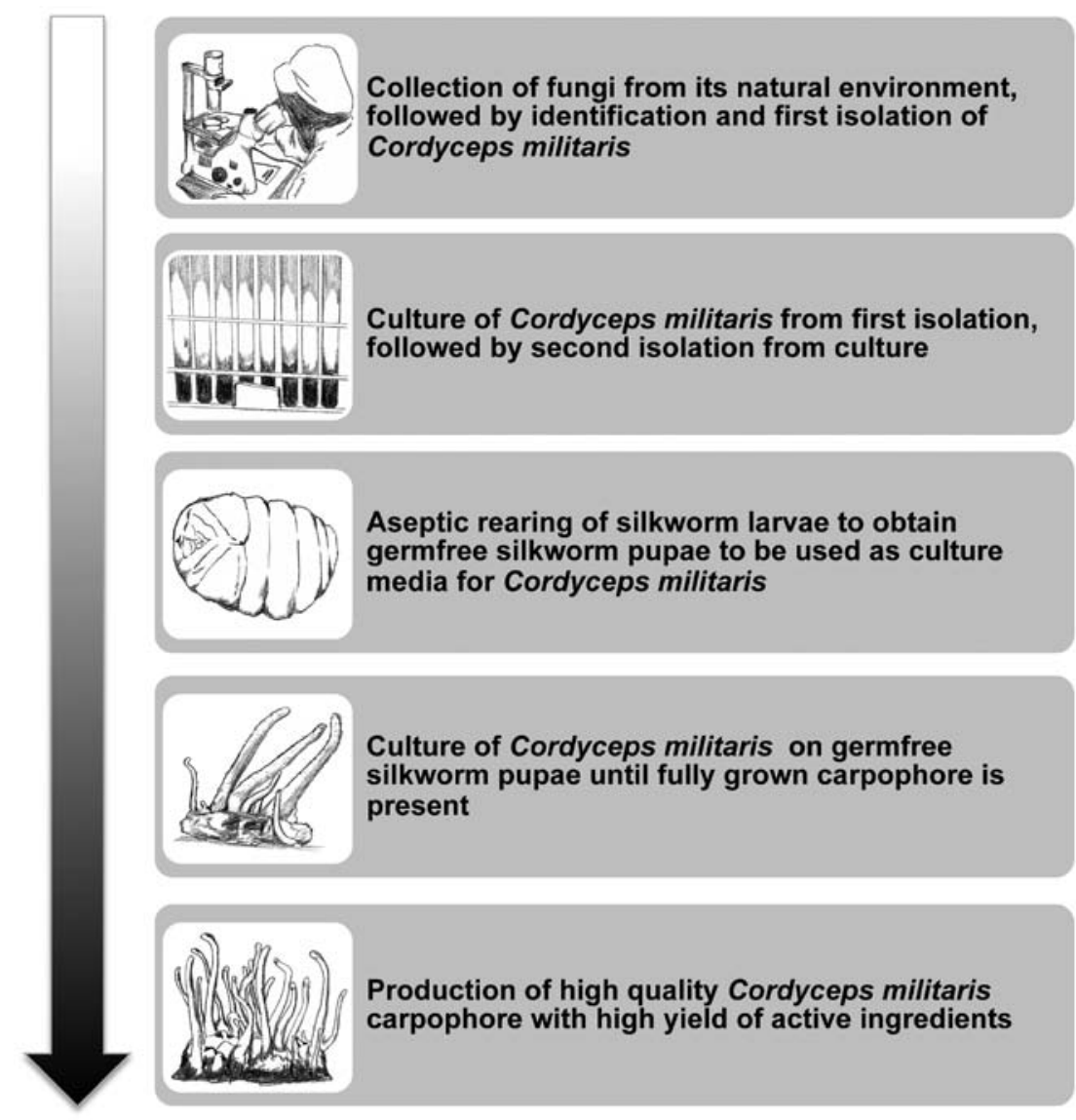

Figure 1. Production of high-quality C. militaris extract. C. militaris collected from wild mushrooms goes through a 2-step isolation process. After culturing the first isolation product, the culture is resampled for a second isolation of C. militaris. The highly purified product of the second isolation is then infected onto germfree silkworm pupae reared through a highly controlled aseptic method. Aseptic rearing of silkworms is highly renewable, assuring production of pupae all year round and also producing other beneficial materials such as germfree silk powder. Active ingredients of $C$. militaris can only be harvested after complete growth of the fungal carpophore.

control group following the same protocol. For comparison, the volume and weight of the subcutaneous tumors were measured in each group. Tumor volumes in each group were calculated every two days as (length $\mathrm{x}$ width $\left.{ }^{2}\right) \mathrm{x} 0.5 \mathrm{~mm}^{3}$.

Statistical analysis. Data are expressed as means \pm SD. We employed simple pair-wise comparison with Student's t-test (two-tailed distribution with two-sample equal variance), and $\mathrm{P}<0.05$ was considered significant.

\section{Results}

Extract of C. militaris has an ability similar to that of synthetic cordycepin for controlling cell growth and inducing apoptosis. A number of culture techniques for this mushroom have been established, for example, storage/stock culture, pre-culture, popular/indigenous culture (spawn culture, husked rice culture and saw dust culture) and special/laboratory culture (shaking culture, submerged culture, surface liquid culture and continuous/repeated batch culture), which vary in places and environment and with aim of the culture (28). We developed a method for isolation and culture of $C$. militaris in order to produce high-quality extract with a high yield of active ingredients under sterilized conditions but economically, and we used it for the purposes of this study (Fig. 1). We evaluated the ability of increasing percentages of $C$. militaris extract in controlling cell growth and apoptosis compared to that of increasing concentrations of synthetic cordycepin. The efficiency of the $C$. militaris extract for controlling MeWo cell growth rate and apoptosis was similar to that of synthetic cordycepin (Fig. 2A-C). For the following experiments, we used $5 \%$ extract of $C$. militaris and $20 \mu \mathrm{M}$ cordycepin to determine the underlying mechanism.

Extract of C. militaris reduces production of VEGF and transcription of VEGF-A and VEGF-B. In order to uncover the role of $C$. militaris extract and cordycepin in angiogenesis, we used a human angiogenesis antibody array. Of note, both $5 \%$ extract and $20 \mu \mathrm{M}$ cordycepin significantly decreased production of angiogenin, VEGF and PDGF-AA. Unexpectedly, $5 \%$ C. militaris extract had a stronger effect on production of VEGF and TIMP-1 compared to cordycepin (Fig. 3A). A higher dose of cordycepin $(100 \mu \mathrm{M})$ reduced the production of VEGF by less than that in the case of $20 \mu \mathrm{M}$ cordycepin, suggesting that a lower dose of cordycepin is required for inhibiting the production of VEGF (Fig. 3A, lowest panel). We also used qRT-PCR analysis to examine whether protein level is associated with mRNA level by $5 \% \mathrm{C}$. militaris extract treatment. Interestingly, not only the mRNA levels of VEGF-A but also that of VEGF-B were significantly reduced 
A
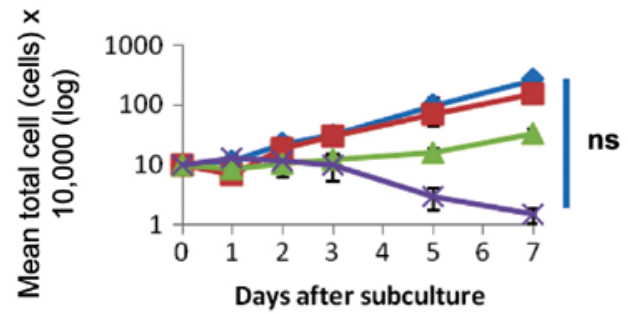

$\leadsto(-)$

$-1 \%$ C. militaris extract

$-5 \%$ C. militaris extract

- $10 \%$ C. militaris extract
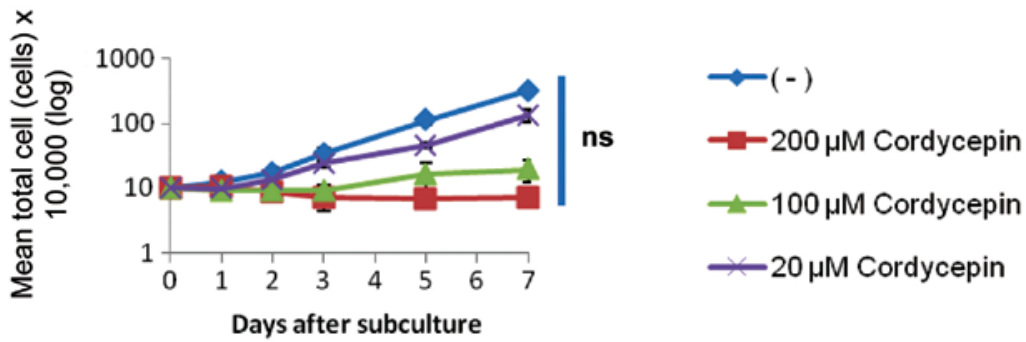

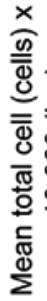
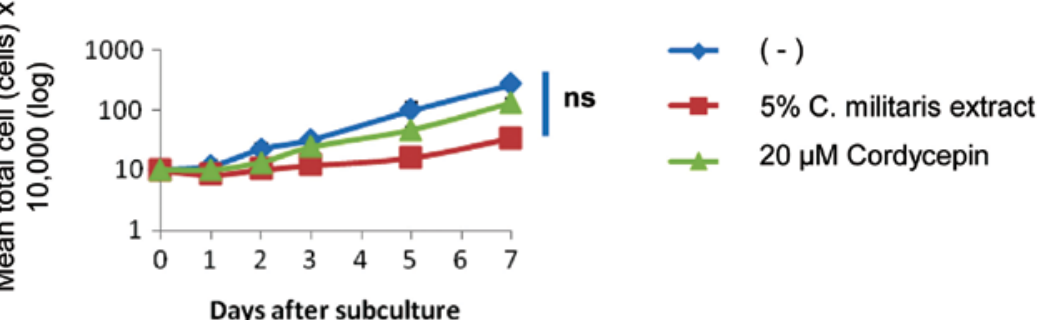

B
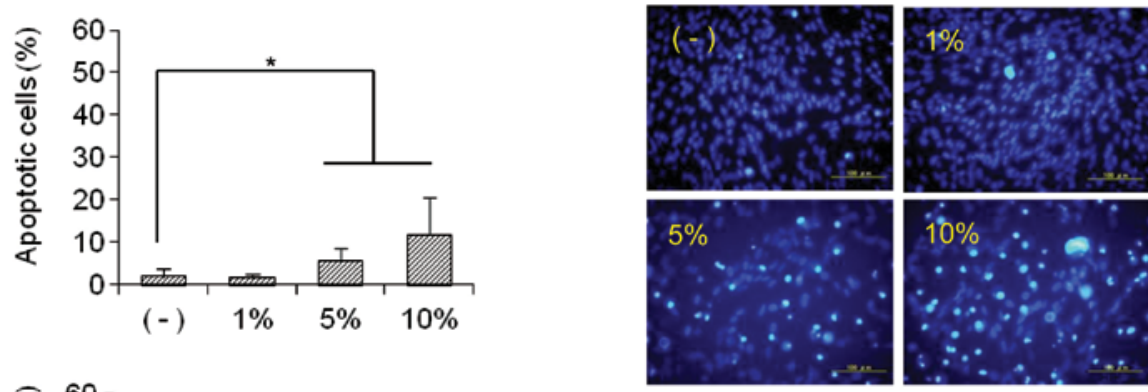

C

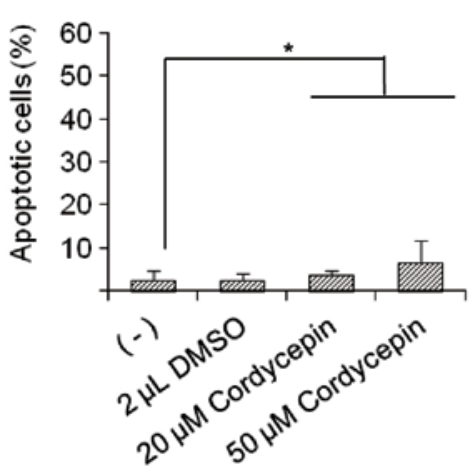

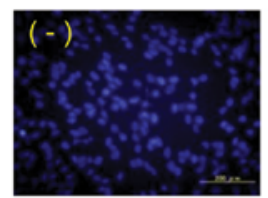

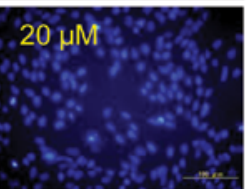

\section{DMSO}

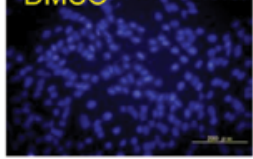

$50 \mu M^{\prime}$

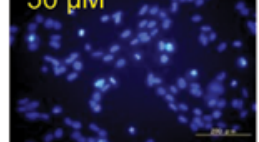

Figure 2. Ability of $C$. militaris to control cell growth and cell apoptosis is comparable to that of synthetic cordycepin. (A) Control of cell growth after treatment with C. militaris extract or cordycepin. MeWo melanoma cells were cultured in groups of triplicates for 7 days. The groups were then treated with increasing concentrations of $C$. militaris extract or cordycepin on the remaining cultures on days 1,3 and 5 . Cell counts using a hemocytometer were performed on days 1,2,3,5 and 7 after seeding. Results suggested that the effect of 5\% C. militaris extract was comparable to that of $20 \mu \mathrm{M}$ cordycepin; ns, not significant. Induction of apoptosis by C. militaris extract (B) or cordycepin (C). MeWo cells were incubated with increasing concentrations of C. militaris extract or cordycepin for $48 \mathrm{~h}$. Apoptotic cells were identified after staining with Hoechst 33342. Representative image (right) and quantified data (left) are shown in (B and C), respectively. Statistically significant differences compared to the control were found in treatment groups of 5 and $10 \%$ C.militaris extract and 20 and $50 \mu \mathrm{M}$ of cordycepin.

by treatment with $C$. militaris extract (Fig. 3B). Treatment with $20 \mu \mathrm{M}$ cordycepin only reduced the mRNA level of VEGF-A (Fig. 3C). The results indicate that $C$. militaris extract has a broader effect than that of cordycepin in controlling angiogenesis, suggesting that other ingredients in the extract reduce the mRNA level of VEGF-B. 


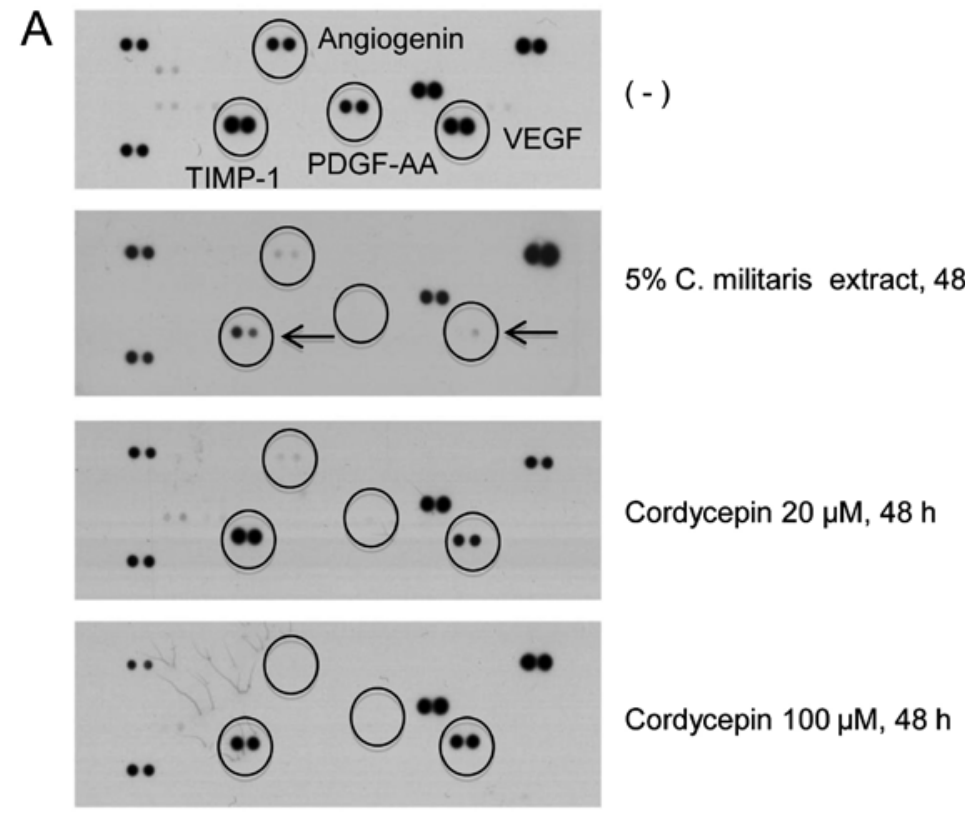

B

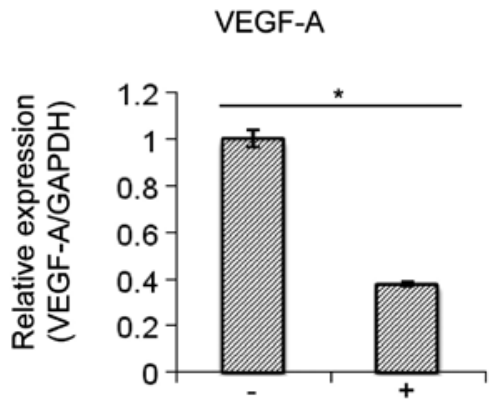

C

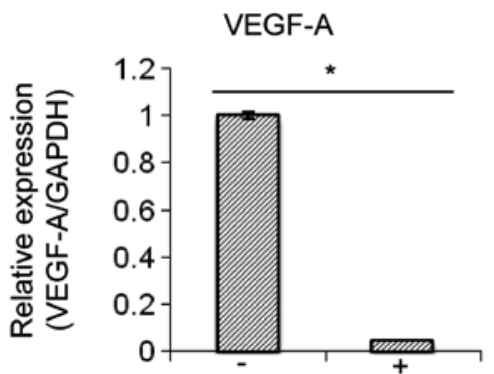

Figure 3. Reduced production of VEGF and transcription of both VEGF-A and VEGF-B by $C$. militaris extract. (A) Dot blot-human angiogenesis array of MeWo culture medium after $C$. militaris extract or cordycepin treatment. Culture media of MeWo cells incubated with control, $5 \%$. militaris extract, $20 \mu \mathrm{M}$ cordycepin, or $100 \mu \mathrm{M}$ cordycepin for $48 \mathrm{~h}$ were used to detect the production of various angiogenesis-related proteins. All treatment groups showed lower production of angiogenin, TIMP-1, PDGF-AA, and VEGF compared to the control group. Production of TIMP-1 and VEGF was especially reduced in the $5 \%$ C. militaris treatment group. (B and C) qPCR of VEGF-A and VEGF-B. Transcription levels of VEGF-A and VEGF-B were quantified from MeWo cells after incubation with either 5\% C. militaris extract (B) or $20 \mu \mathrm{M}$ cordycepin (C) for $48 \mathrm{~h}$. Transcription levels of VEGF-A and VEGF-B were significantly decreased after treatment with $5 \%$ C. militaris extract, while only VEGF-A was decreased after treatment with $20 \mu \mathrm{M}$ cordycepin. ${ }^{*} \mathrm{P}<0.05$ significantly different from the control group. ns, not significant.

Extract of $C$. militaris reduces the production of VEGF through downregulation of Akt1 and GSK $3 \alpha / 3 \beta$. In order to elucidate the molecular mechanism underlying the properties of $C$. militaris extract, we used a human phospho-MAPK array. The extract of $C$. militaris reduced Akt1 and GSK $3 \alpha / 3 \beta$ levels but increased p38 $\alpha$ level (Fig. 4A). Furthermore, we investigated the roles of PI $3 \mathrm{~K}$, Akt1, GSK-3 $\beta$ and $\mathrm{p} 38 \alpha$, in regulating mRNA levels of VEGF-A and VEGF-B by qRT-PCR in human MM cells (MeWo) transiently overexpressing the aforementioned protein kinases. The messenger RNA level of VEGF-A was upregulated by cells overexpressing p38 $\alpha$, PI3K and Akt1. The mRNA level of VEGF-B was upregulated by cells overexpressing p38 $\alpha$, GSK-3 $\beta$, PI3K and Akt1. GSK-3 $\beta$ showed specificity for regulating the mRNA of VEGF-B, suggesting that the additional effect of $C$. militaris extract treatment in decreasing VEGF-B was at least in part due to 

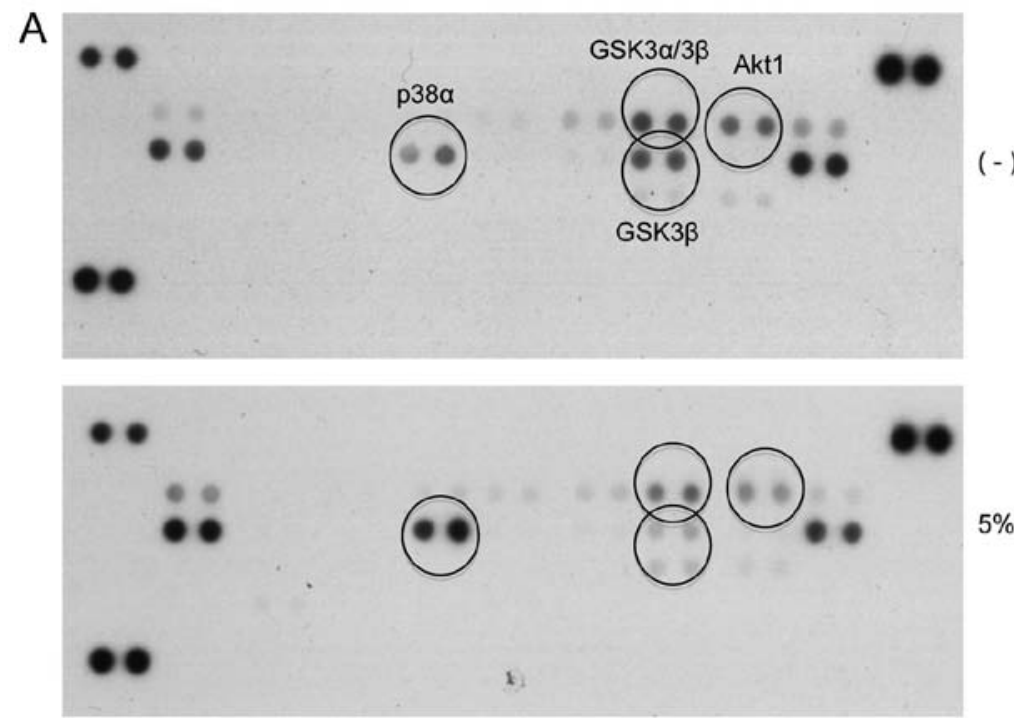

$5 \%$ C. militaris extract, $48 \mathrm{~h}$

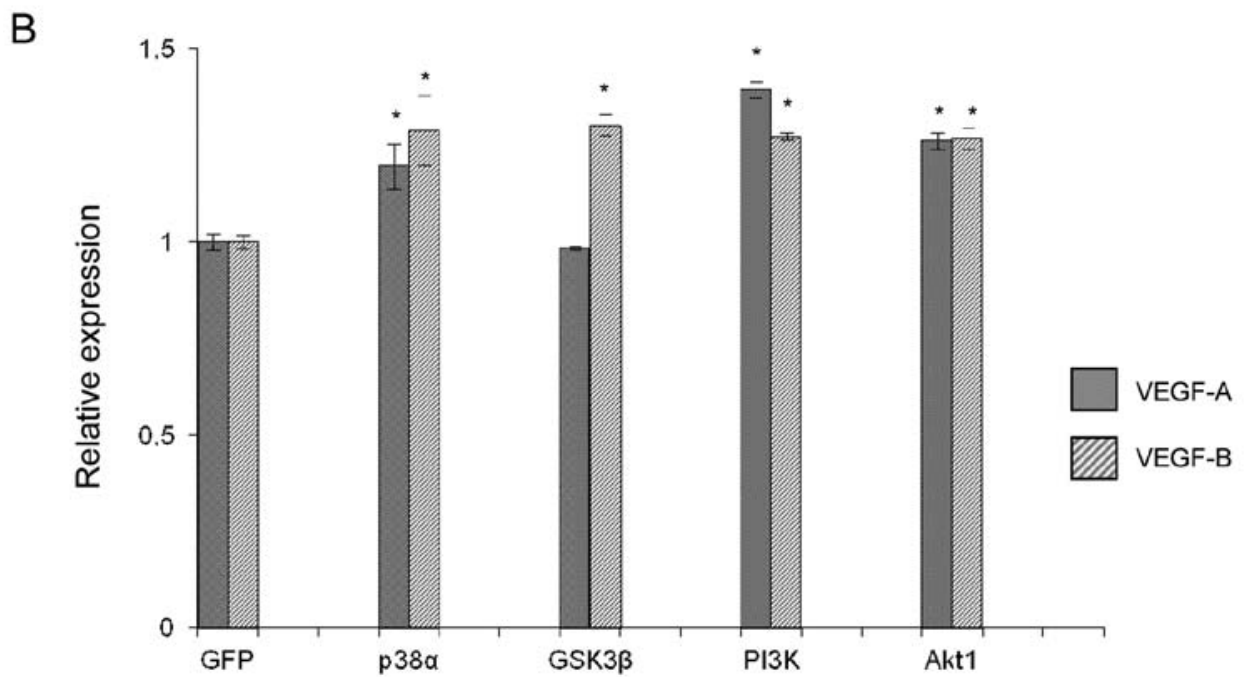

Figure 4. Reduced production of VEGF by $C$. militaris extract was related to the downregulation of GSK $3 \alpha / 3 \beta$ and Akt1. (A) Phospho-MAPK array of MeWo cell lysate after treatment with control or 5\% C. militaris extract. Cell lysate from MeWo cells incubated with control or 5\% C. militaris extract for $48 \mathrm{~h}$ showed phosphorylation of several protein kinases, particularly p $38 \alpha$, GSK $3 \alpha / 3 \beta$, and Akt1. Addition of $5 \%$ C. militaris extract resulted in an increase in phosphorylation of p38 $\alpha$ but decrease of GSK $3 \alpha / 3 \beta$ and Akt1. (B) qRT-PCR of VEGF-A and VEGF-B mRNA levels in MeWo cells overexpressing p38 $\alpha$, GSK3 $\beta$, PI3K, and Akt1. MeWo cells overexpressing p38 $\alpha$, GSK3 $\beta$, PI3K or Akt1 showed a significant increase of VEGF-B mRNA levels, while VEGF-A mRNA was only found to significantly increase in cells overexpressing p38, PI $3 \mathrm{~K}$, and Akt1, indicating the possibility that $5 \%$ C. militaris extract lowered VEGF-B phosphorylation levels through GSK3 $\beta$. " $\mathrm{P}<0.05$ significantly different from the control group.

its ability to decrease phosphorylation of GSK-3ß. Moreover, Akt1 upregulated mRNA of VEGF-A and VEGF-B to similar levels (Fig. 4B), indicating a possible role of C. militaris extract in the Akt/ GSK-3ß pathway.

In vivo growth retardation of subcutaneously implanted melanocytic tumors by $C$. militaris extract. A mouse model of a xenografted melanocytic tumor was generated by subcutaneous injection of a human MM cell line (MeWo) in the flank region of Balb/c nude mice. The tumor cells gradually grew at the implanted site, forming a visual tumor mass after 3-4 weeks. When the tumors had grown to $\sim 100 \mathrm{~mm}^{3}$ in each mouse, the mice were divided into two groups, which were treated with or not treated with subcutaneous administration of $C$. militaris extract (vehicle: saline only) once every two days for 37 days consecutively. At day 37 after the initial treatment, tumor size in the $C$. militaris extract-treated group was significantly smaller than that in the untreated group (Fig. 5A). Histological examination of the excised melanocytic tumors from mice treated with $C$. militaris extract showed massive necrotic/degenerative lesions inside the tumors, which were observed less in tumors of the untreated mice group (Fig. 5B, upper panel). In immunohistochemical analysis with anti-VEGF antibody, expression of VEGF on tumor cells was diffusely and abundantly observed in untreated tumors, whereas tumor cells lacking VEGF expression or with weak expression were detected in tumors from C. militaris extract-treated mice (Fig. 5B, left lower panel). Moreover, anti-mouse CD31 staining revealed 

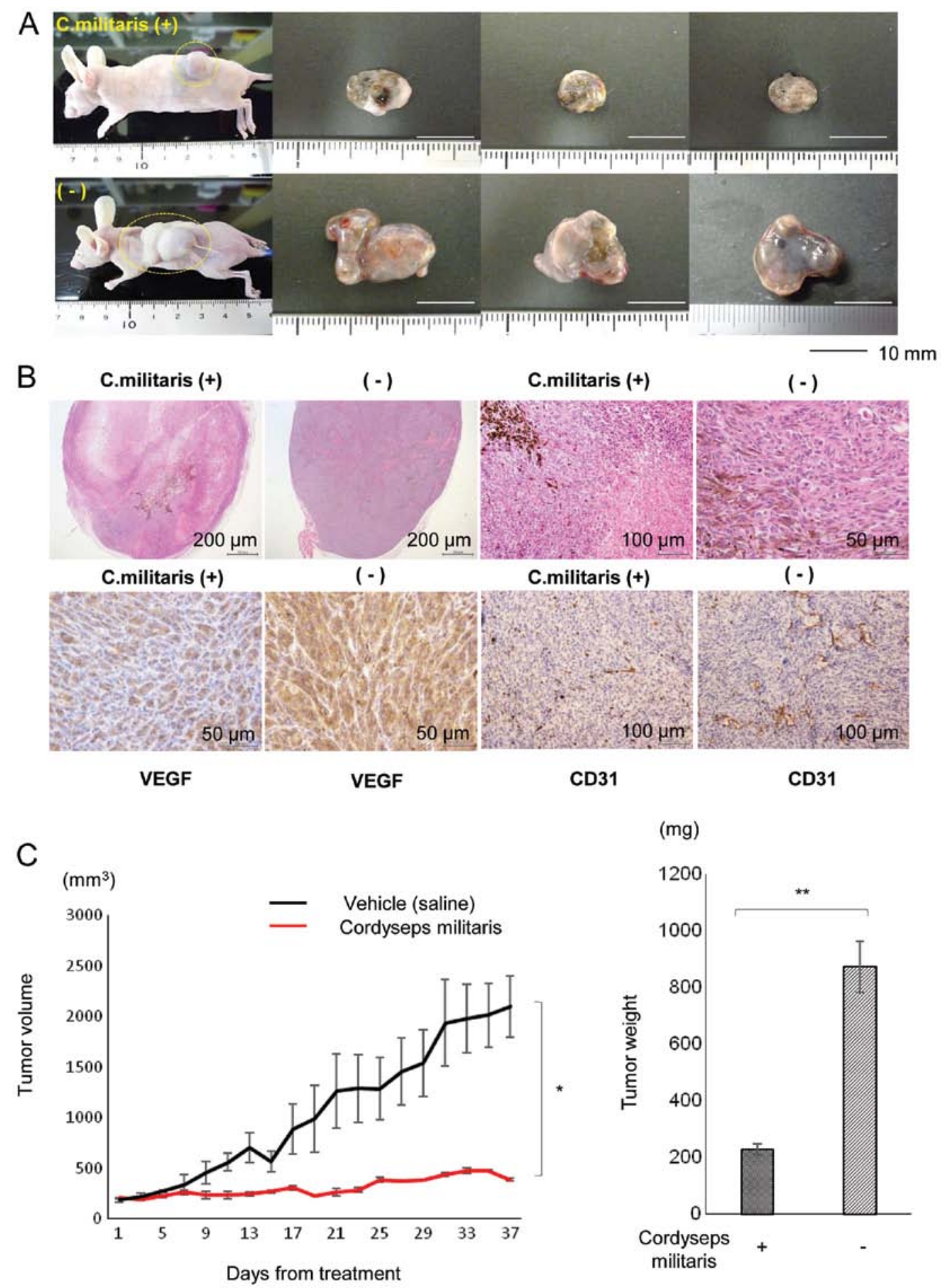

Figure 5. Treatment with C. militaris extract reduced growth of subcutaneously xenografted melanomas. (A) Gross appearance of xenografted tumors. Tumors with subcutaneous melanoma tissues were excised from mice after 37 days with or without $C$. militaris extract treatment. Compared to the control group, C. militaris-treated mice showed small tumors. (B) Histology (H\&E staining) and immunohistochemistry of excised tumors of mice treated with C. militaris or saline (control). Expression of VEGF on tumor cells and morphology of vascular vessels in tumor tissues stained by CD31 mAb are shown. Both VEGF and CD31 expression were reduced in C. militaris-treated tumors. (C) Growth of subcutaneously xenografted melanomas in mice after treatment with C. militaris or saline. Mice bearing subcutaneous MeWo xenografts were treated with saline or the supernatant of C. militaris after tumor volumes had reached $\sim 100 \mathrm{~mm}^{3}$ (day 1). Tumor-bearing mice in each group were subjected to subcutaneous administration of the supernatant or saline once every two days for 37 days consecutively. Tumor volumes were measured daily from day 1 to day 37 . Statistical significance of tumor volumes and that of tumor masses were evaluated by Student's t-tests. Saline ( $\mathrm{n}=3$, mean volume of $2101 \mathrm{~mm}^{3}$ on day 37) vs. C. militaris $\left(\mathrm{n}=3\right.$, mean volume $384 \mathrm{~mm}^{3}$ on day 37); ${ }^{*} \mathrm{P}=0.069>0.05$. Final masses of tumors excised on day 37 in each group are also shown in the graph (right). Saline ( $\mathrm{n}=3$, mean weight of $874 \mathrm{mg})$ vs. C. militaris $(\mathrm{n}=3$, mean weight of $230 \mathrm{mg})$; ${ }^{* *} \mathrm{P}=0.031<0.05$. Means and $\mathrm{SE}$ (standard error) of triplicates are shown.

undeveloped small blood vessels with thin walls in many of the $C$. militaris extract-treated tumors, whereas the tumors in the untreated mice had developed well-forked vessels with prominent lumens (Fig. 5B, right lower panel). These results were in line with results of the Directed In Vivo Angiogenesis Assay (DIVAA) (Fig. 6A) and chorioallantoic membrane assay 

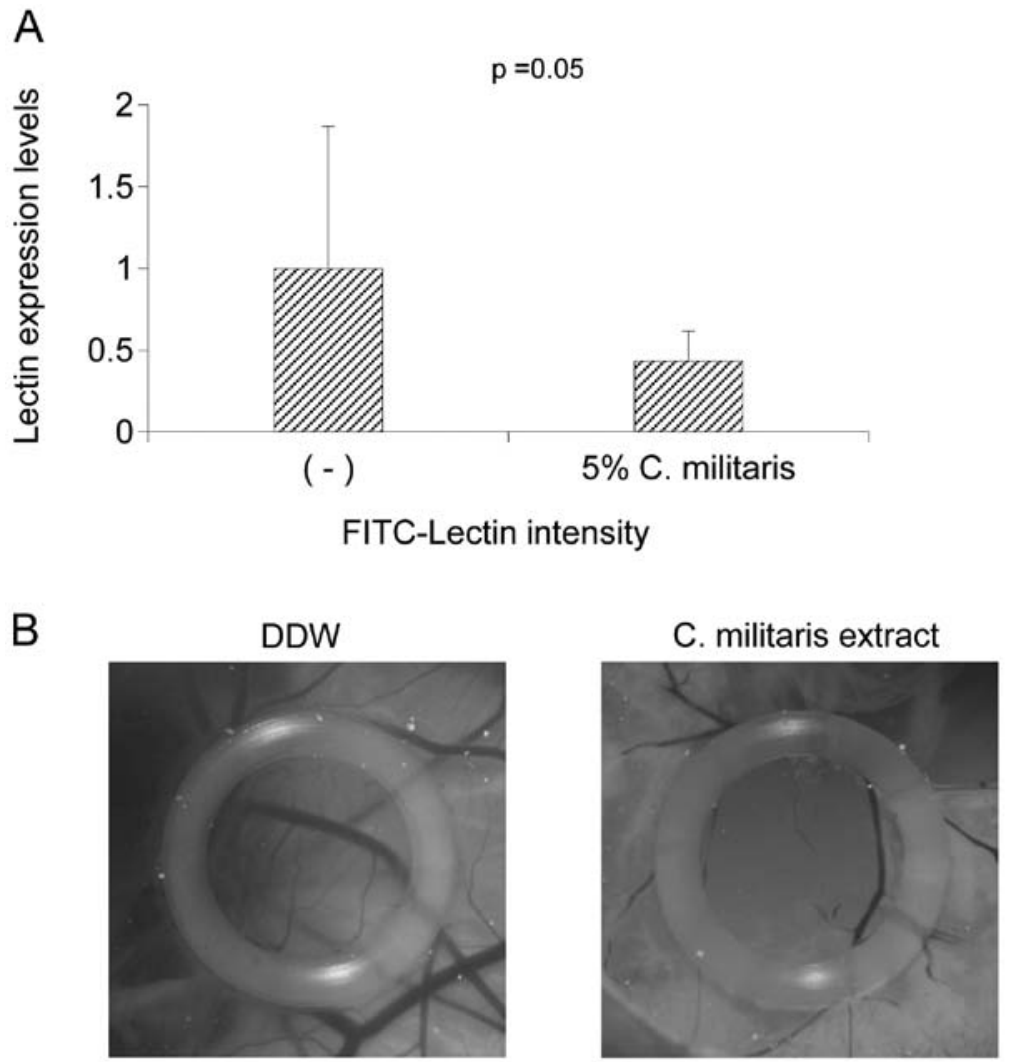

Figure 6. Treatment with C. militaris extract reduced angiogenesis. (A) Decrease of FITC-lectin intensity after treatment with 5\% C. militaris extract. Using the Cultrex Directed In Vivo Angiogenesis Assay (DIVAA) (Trevigen, Gaithersburg, MD, USA), angioreactors containing a mixture of BME and DDW or BME and 5\% C. militaris extract were implanted subcutaneously into the flanks of mice for 16 days. After extraction, FITC-lectin intensity was measured using excitation $485 \mathrm{~nm}$, emission $510 \mathrm{~nm}$. (B) Chorioallantoic membrane (CAM) assay showed that neovascularization was inhibited by C. militaris extract. Eight days post-fertilized chicken egg shells were cut to place silicon rings on the CAM. The inner spaces of the silicon rings were filled with DDW or $50 \mu 1$ of C. militaris extract. Egg shells were covered using transparent tape, and eggs were incubated for $48 \mathrm{~h}$. Results were observed by decrease or increase of vascular branch formation from main capillary vessels.

(CAM) (Fig. 6B) that revealed decreased angiogenic activity by treatment with $C$. militaris extract. Consequently, the tumors derived from $C$. militaris extract-treated mice were less than one-fifth in volume and approximately one-fourth in weight of those of untreated mice, indicating an antitumor effect of $C$. militaris extract for MM-derived tumors in vivo (Fig. 5C).

\section{Discussion}

In this study, we found that $C$. militaris extract, which was isolated and cultured by a newly developed method under sterilized conditions, has an anti-angiogenic property by decreasing the production of VEGF from a human MM cell line (MeWo). We showed that treatment with 5\% C. militaris extract reduced the mRNA levels of VEGF-A and VEGF-B and production of VEGF protein in MeWo cells. Furthermore, we demonstrated that tumor growth in the mouse model was significantly decreased by $C$. militaris extract treatment with reduced vascular density observed. For many tumors, vascular density is a prognostic indicator of metastatic potential, with highly vasculated primary tumors having a higher incidence of metastasis than that of poorly vasculated tumors (4).

Several important signaling pathways, including mitogen-activating protein kinase (MAPK)/extracellular signal-regulated kinase (ERK) and phosphoinositol 3-kinase
(PI3K)/Akt, have been found to be induced by angiogenic growth factors in endothelial cells (29). Activation of these pathways can promote migration, proliferation, differentiation and survival of endothelial cells (30-32). Activation of the PI3K/Akt pathway in tumor cells can also increase VEGF production, by both hypoxia-inducible factor-1 (HIF-1)dependent and independent mechanisms $(33,34)$. The PI3K/Akt pathway also modulates expression of other angiogenic factors such as nitric oxide and angiopoietins (35).

In order to elucidate the molecular mechanism by which C. militaris extract affects the production of VEGF in MeWo cells, we detected reduction of protein kinase GSK $3 \alpha / 3 \beta$ and Akt1 but increase of p38 $\alpha$ by $C$. militaris extract treatment. GSK $3 \beta$ is known to be involved in a variety of cellular processes including glycogen metabolism, insulin signaling, cell proliferation, apoptosis, neuronal function and embryonic development (36-38). When GSK3 $\beta$ is phosphorylated by Akt, the kinase activity is lost, i.e., GSK3 $\beta$ is negatively regulated by Akt. In accordance with our results, Tekle et al reported that treatment of non-small cell lung cancer (NSCLC) with a PKC- $\beta$ inhibitor increased apoptosis and reduced Akt phosphorylation followed by activation of GSK $3 \beta$, and mitigation of VEGF production (39). Graff et al (40), Hanauske et al (41), and Lee et al (42) also reported inhibition of tumor growth by a PKC- $\beta$ inhibitor through marked reduction of VEGF production in breast, thyroid, head/neck, non-small cell lung cancer, 
pancreatic cancer and melanoma cancer cell lines and also in patient-derived tumor explants. This suggests a possible involvement of PKC- $\beta$ in VEGF production through a regulation of Akt-GSK3 $\beta$ pathway. Further investigation is needed to clarify the mechanism.

In this study, we also found a decrease in Akt1 level induced by treatment with $C$. militaris extract. Akt plays an important role in both physiological and pathological angiogenesis through effects in both endothelial cells and cells producing angiogenic signals, such as tumor cells (43). Akt1 is the predominant isoform ubiquitously expressed in a variety of tissues and endothelial cells (44). Akt1 is vital for the regulation of vascular permeability, angiogenic responses and subsequent vascular maturation $(45,46)$. Akt1 is necessary for inside-out integrin signaling. Impaired integrin function often results in decreased extracellular matrix assembly and deposition; this, in turn, might affect the integrity of the endothelial monolayer and its permeability, an essential part of the angiogenic response $(47,48)$. Therefore, we suggest that angiogenesis in the tumor-bearing mice was perturbed by treatment with $C$. militaris extract, which further lead to a decrease in the supply of nutrition or growth factors required by tumor cells to proliferate.

A recent study exploring the role of MAPK and PI3K signaling in angiogenesis of squamous cell carcinoma of the head and neck revealed that EGFR activation induces VEGF-A expression that requires both PI3K and MAPK (49). p38 MAP kinases (p38) are activated by extracellular or intracellular stresses $(50,51)$. and lipopolysaccharide (LPS) (52), and thus their activation generally promotes growth inhibition and/or induces apoptosis $(50,53)$. Our study showed an increase in $\mathrm{p} 38 \alpha$ upon treatment with $C$. militaris extract, suggesting additional inhibition of tumor growth through apoptotic induction, which supports its anti-angiogenic properties.

Despite accumulating evidence indicating an important role of VEGF signaling in angiogenesis, we realize that there are many other angiogenic pathways with observable effects on vessel growth in vivo that have not been completely characterized. These include chemokines, integrins (54), several transcriptional regulators, Wnt ligands and their frizzled receptors (55), other members of the FGF, PDGF and TGF- $\beta$ superfamilies, and the VEGF homolog PIGF that transmit angiogenic signals through VEGFR1 (56). Identifying their role in vessel branching or other types of vessel growth will lead to the generation of a better comprehensive model that can serve as a source for future drug development. In this study, we focused on the role of $C$.militaris extract in affecting VEGF production of a human MM cell line in angiogenesis and tumor growth. Further studies are required to enhance the understanding of a complex angiogenic pathway, especially other angiogenic pathways independent of VEGF.

In conclusion, the extract of $C$. militaris that we isolated and cultured by a newly developed method is a very promising anticancer agent. It has properties targeting several cellular signaling pathways that are involved in induction of apoptosis and inhibition of the angiogenesis of tumor cells. These properties were mediated by reducing GSK $3 \beta$ and Akt1 phosphorylation, which led to decreased VEGF production and increased phosphorylation of $\mathrm{p} 38 \alpha$ that induced apoptosis. These results make the effect of $C$. militaris a good candidate for different combination regimens, including combinations such as pre-treatment or co-treatment with other novel targeting agents and cytotoxic drugs commonly used in a clinical setting.

\section{Acknowledgements}

This study was supported in part by grants from the Ministry of Education, Culture, Sports, Science and Technology of Japan (Grant-in-Aid for Scientific Research on Innovation Areas, no. 24117711) (M. Sakaguchi).

\section{References}

1. Folkman J: Angiogenesis in cancer, vascular, rheumatoid and other disease. Nat Med 1: 27-31, 1995.

2. Dewing D, Emmett M and Pritchard Jones R: The roles of angiogenesis in malignant melanoma: trends in basic science research over the last 100 years. ISRN Oncol 2012: 546927, 2012.

3. Folkman J: Role of angiogenesis in tumor growth and metastasis. Semin Oncol 29: 15-18, 2002.

4. Zetter BR: Angiogenesis and tumor metastasis. Annu Rev Med 49: 407-424, 1998 .

5. Naumov GN, Bender E, Zurakowski D, et al: A model of human tumor dormancy: an angiogenic switch from the non-angiogenic phenotype. J Natl Cancer Inst 98: 316-325, 2006.

6. Nanus DM, Schmitz-Dräger BJ, Motzer RJ, et al: Expression of basic fibroblast growth factor in primary human renal tumors: correlation with poor survival. J Natl Cancer Inst 85: 1597-1599, 1993.

7. Toi M, Hoshina S, Takayanagi $\mathrm{T}$ and Tominaga $\mathrm{T}$ : Association of vascular endothelial growth factor expression with tumor angiogenesis and with early relapse in primary breast cancer. Jpn J Cancer Res 85: 1045-1048, 1994.

8. Relf M, LeJeune S, Scott PA, et al: Expression of the angiogenic factors, vascular endothelial growth factor, acidic and basic fibroblast growth factor, tumor growth factor beta-1, platelet derived growth endothelial cell growth factor, placental growth factor, and pleiotrophin in human primary breast cancer and its relation to angiogenesis. Cancer Res 57: 963-969, 1997.

9. Kandel J, Bossy-Wetzel E, Radvanyi F, Klagsbrun M, Folkman J and Hanahan D: Neovascularization is associated with a switch to the export of bFGF in the multistep development of fibrosarcoma. Cell 66: 1095-1104, 1991.

10. Hanahan D and Folkman J: Patterns and emerging mechanisms of the angiogenic switch during tumorigenesis. Cell 86: 353-364, 1996.

11. Ferrara N: Vascular endothelial growth factor. Eur J Cancer 32A: 2413-2422, 1996.

12. Risau W: Mechanisms of angiogenesis. Nature 386: 671-674, 1997.

13. Shibuya M: Vascular endothelial growth factor-dependent and independent regulation of angiogenesis. BMB Rep 41: 278-286, 2008.

14. Benjamin LE and Keshet E: Conditional switching of vascular endothelial growth factor (VEGF) expression in tumors: induction of endothelial cell shedding and regression of hemangioblastoma-like vessels by VEGF withdrawal. Proc Natl Acad Sci USA 94: 8761-8766, 1997.

15. Shibuya M: Differential roles of vascular endothelial growth factor receptor-1 and receptor-2 in angiogenesis. J Biochem Mol Biol 39: 469-478, 2006.

16. Ellis LM and Hicklin DJ: VEGF-targeted therapy: mechanisms of anti-tumour activity. Nat Rev Cancer 8: 579-591, 2008.

17. Dvorak HF: Vascular permeability factor/vascular endothelial growth factor: a critical cytokine in tumor angiogenesis and a potential target for diagnosis and therapy. J Clin Oncol 20: 4368-4380, 2002.

18. Erhard H, Rietveld FJ, van Altena MC, Bröcker EB, Ruiter DJ and de Waal RM: Transition of horizontal to vertical growth phase melanoma is accompanied by induction of vascular endothelial growth factor expression and angiogenesis. Melanoma Res 7 (Suppl 2): S19-S26, 1997.

19. Bergers $G$ and Hanahan D: Modes of resistance to anti-angiogenic therapy. Nat Rev Cancer 8: 592-603, 2008. 
20. Potente M, Gerhardt $\mathrm{H}$ and Carmeliet P: Basic and therapeutic aspects of angiogenesis. Cell 146: 873-887, 2011.

21. Cunningham KG, Hutchinson SA, Manson W and Spring FS: Cordycepin, a metabolic product from cultures of $C$. militaris (Linn.) Link. Part I. Isolation and characterisation. J Chem Soc: 508: 2299-3200, 1951.

22. Song CH, Jeon YJ, Yang BK, Ra KS and Sung JM: Anticomplementary activity of exo-polymers produced from submerged mycelial cultures of higher fungi with particular reference to Cordyceps militaris. J Microbiol Biotechnol 8: 536-539, 1998

23. Mizuno T: Medicinal effects and utilization of Cordyceps (Fr.) Link (Ascomycetes) and Isaria Fr. (Mitosporic Fungi) Chinese caterpillar fungi, 'Tochukaso' (Review). Int J Med Mushroom 1: 251-261, 1999.

24. Nag TB and Wang HX: Pharmacological actions of Cordyceps, a prized folk medicine. J Pharm Pharmacol 57: 1509-1519, 2005.

25. Das SK, Masuda M, Hatashita M, Sakurai A and Sakakibara M: A new approach for improving cordycepin productivity in surface liquid culture of $C$. militaris using high-energy ion beam irradiation. Lett Appl Microbiol 47: 534-538, 2008.

26. Das SK, Masuda M, Sakurai A and Sakakibara M: Medicinal uses of the mushroom Cordyceps militaris: current state and prospects. Fitoterapia 8: 961-968, 2010.

27. Sakaguchi M, Huh N and Namba M: A novel tumor suppressor, REIC/Dkk-3 gene identified by our in vitro transformation model of normal human fibroblasts works as a potent therapeutic antitumor agent. Adv Exp Med Biol 720: 209-215, 2012.

28. Das SK, Masuda M, Hatashita M, Sakurai A and Sakakibara M Optimization of culture medium for cordycepin production using $C$. militaris mutant obtained by ion beam irradiation. Proc Biochem 45: 129-132, 2010.

29. Graupera M and Potente M: Regulation of angiogenesis by PI3K signaling networks. Exp Cell Res 319: 1348-1355, 2013.

30. Graupera M, Guillermet-Guibert J, Foukas LC, et al Angiogenesis selectively requires the p110 alpha isoform of PI3K to control endothelial cell migration. Nature 453: 662-666, 2008.

31. Wang F, Yamauchi M, Muramatsu M, Osawa T, Tsuchid R and Shibuya M: MRACK1 regulates VEGF/Flt1-mediated cell migration via activation of a PI3K/Akt pathway. J Biol Chem 286: 9097-9106, 2011.

32. Zhao P, Meng Q, Liu LZ, You YP, Liu N and Jiang BH: Regulation of survivin by PI3K/Akt/p70S6K1 pathway. Biochem Biophys Res Commun 395: 219-224, 2010.

33. Maity A, Pore N, Lee J, Solomon D and O'Rourke DM: Epidermal growth factor receptor transcriptionally up-regulates vascular endothelial growth factor expression in human glioblastoma cells via a pathway involving phosphatidylinositol 3-kinase and distinct from that induced by hypoxia. Cancer Res 60: 5879-5886, 2000.

34. Zhong H, Chiles K, Feldse D, et al: Modulation of hypoxiainducible factor 1alpha expression by the epidermal growth factor/phosphatidylinositol3-kinase/PTEN/AKT/FRAP pathway in human prostate cancer cells: implications for tumor angiogenesis and therapeutics. Cancer Res 60: 1541-1545, 2000.

35. Karar J and Maity A: PI3K/AKT/mTOR pathway in angiogenesis Front Mol Neurosci 4: 51, 2011.

36. Grimes CA and Jope RS: The multifaceted roles of glycogen synthase kinase $3 \beta$ in cellular signaling. Prog Neurobiol 65: 391-426, 2001.

37. Forde JE and Dale TC: Glycogen synthase kinase 3: a key regulator of cellular fate. Cell Mol Life Sci 64: 1930-1944, 2007.

38. Rayasam GV, Tulasi VK, Sodhi R, Davis JA and Ray A: Glycogen synthase kinase 3: more than a namesake. Br J Pharmacol 156 : 885-898, 2009.
39. Tekle C, Giovannetti E, Sigmond J, Graff JR, Smid K and Peters GJ: Molecular pathways involved in the synergistic interaction of the PKC $\beta$ inhibitor enzastaurin with the antifolate pemetrexed in non-small cell lung cancer cells. Br J Cancer 99: 750-759, 2008

40. Graff JR, McNulty AM, Hanna KR, et al: The protein kinase Cbeta-selective inhibitor, Enzastaurin (LY317615.HCl), suppresses signaling through the AKT pathway, induces apoptosis, and suppresses growth of human colon cancer and glioblastoma xenografts. Cancer Res 65: 7462-7469, 2005.

41. Hanauske AR, Oberschmidt O, Hanauske-Abel H, Lahn MM and Eismann U: Antitumor activity of enzastaurin (LY317615.HCl) against human cancer cell lines and freshly explanted tumors investigated in in-vitro (corrected) soft-agar cloning experiments. Invest New Drugs 25: 205-210, 2007.

42. Lee KW, Kim SG, Kim HP, et al: Enzastaurin, a protein kinase C beta inhibitor, suppresses signaling through the ribosomal S6 kinase and bad pathways and induces apoptosis in human gastric cancer cells. Cancer Res 68: 1916-1926, 2008.

43. Manning BD and Cantley LC: AKT/PKB signaling: navigating downstream. Cell 129: 1261-1274, 2007.

44. Kandel ES and Hay N: The regulation and activities of the multifunctional serine/threonine kinase Akt/PKB. Exp Cell Res 253: 210-229, 1999.

45. Chen J, Somanath PR, Razorenova OV, Chen WS, Hay N, Bornstein P and Byzova TV: Aktl regulates pathological angiogenesis, vascular maturation and permeability in vivo. Nature Med 11: 1188-1196, 2005.

46. Somanath PR, Razorenova OV, Chen J and Byzova TV: Akt1 in Endothelial cell and angiogenesis. Cell Cycle 5: 512-518, 2006.

47. Ruoslahti E and Engvall E: Integrins and vascular extracellular matrix assembly. J Clin Invest 99: 1149-1152, 1997.

48. Carmeliet P, Moons L, Luttun A, et al: Synergism between vascular endothelial growth factor and placental growth factor contributes to angiogenesis and plasma extravasation in pathological conditions. Nat Med 7: 575-583, 2001

49. Luangdilok S, Box C, Harrington K, Rhys-Evans P and Eccles S: MAPK and PI3K signalling differentially regulate angiogenic and lymphangiogenic cytokine secretion in squamous cellcarcinoma of the head and neck. Eur J Cancer 47: 520-529, 2011.

50. Kyriakis JM and Avruch J: Sounding the alarm: Protein kinase cascades activated by stress and inflammation. J Biol Chem 271: 24313-24316, 1996.

51. Conrad PW, Rust RT, Han J, Millhorn DE and Beitner-Johnson D: Selective activation of p38alpha and p38gamma by hypoxia. Role in regulation of cyclin D1 by hypoxia in PC12 cells. J Biol Chem 274: 23570-23576, 1999.

52. Lai WC, Zhou M, Shankavaram U, Peng G and Wahl LM: Differential regulation of lipopolysaccharide induced monocyte matrix metalloproteinase (MMP)-1 and MMP-9 by p38 and extracellular signal-regulated kinase $1 / 2$ mitogen-activated protein kinases. J Immunol 170: 6244-6249, 2003.

53. Xia Z, Dickens M, Raingeaud J, Davis RJ and Greenberg ME: Opposing effects of ERK and JNK-p38 MAP kinases on apoptosis. Science 270: 1326-1331, 1995.

54. De S, Razorenova O, McCabe NP, O'Toole T, Qin J and Byzova TV: VEGF-integrin interplay controls tumor growth and vascularization. Proc Natl Acad Sci USA 102: 7589-7594, 2005.

55. Franco CA, Liebner S and Gerhardt H: Vascular morphogenesis: a Wnt for every vessel? Curr Opin Genet Dev 19: 476-483, 2009.

56. Fischer C, Mazzone M, Jonckx B and Carmeliet P: FLT1 and its ligands VEGFB and PIGF: drug targets for anti-angiogenic therapy? Nature Rev Cancer 8: 942-956, 2008. 